\title{
Selective Nanomechanics of Aromatic versus Aliphatic Thiolates on Gold Surfaces
}

\author{
Martin E. Zoloff Michoff, ${ }^{*}$ Jordi Ribas-Arino, ${ }^{\dagger}$ and Dominik Marx \\ Lehrstuhl für Theoretische Chemie, Ruhr-Universität Bochum, 44780 Bochum, Germany
}

(Received 23 May 2018; revised manuscript received 23 December 2018; published 25 February 2019)

\begin{abstract}
Thiolated gold nanointerfaces play a key role in numerous fields of science, technology, as well as modern medicine to coat, functionalize, and protect. Our computational study reveals that the mechanical vs thermal stabilities of aliphatic thiolates on gold surfaces are strikingly different from those of aromatic thiolates. The aliphatic thiolates feature, at the same time, a higher thermal desorption energy but a lower mechanical rupture force than thiophenolates. Our analysis discloses that this most counterintuitive property is due to different mechanochemical detachment mechanisms. Electronic structure analyses along the detachment pathways trace this back to the distinct electronic properties of the S-Au bond in stretched nanojunctions. The discoveries that it is a higher thermal stability that entails a lower mechanical stability and that mechanical loads generate different local nanostructures depending on the nature of the thiolate are highly relevant for the rational design of improved thiol-gold nanocontacts.
\end{abstract}

DOI: 10.1103/PhysRevLett.122.086801

The interest in gold-based hybrid organic-inorganic interfacial materials has dramatically increased over the past decade due to their potential applications in diverse fields such as heterogeneous catalysis [1-3], molecular electronics [4-8], nanophotonics [9], as well as molecular biology and biomedicine [10-15]. Many of these applications involve the tailored modification of the metallic surface by incorporating molecules that provide new properties to the hybrid interface. In particular, due to the strength of the $\mathrm{S}-\mathrm{Au}$ bond, thiolates on gold have been widely used for this purpose [16-20]. For instance, the interactions with protecting thiols can be used to tune the size [21], shape [22], and crystalline phase [23] of gold nanoclusters, $\operatorname{Au}_{n}(\mathrm{SR})_{m}$ [24-27]. The understanding of the S-Au interaction [28-32], thus, becomes highly relevant from an application point of view. Still, not much systematic knowledge is available when it comes to predicting the impact of the organic molecule on such properties.

In this realm, mechanical stability towards friction and wear is critical, yet it is often neglected in the design of molecule-based devices at the nanometer scale. However, force-induced degradation and detachment processes are key aspects for self-assembled monolayers and molecular nanojunctions of various alkyl thiolates on noble metals (see Sec. 4.1 of Ref. [33] and Refs. [28,34-38]).

Surprisingly, aromatic thiolate ligands have received much less attention [39-45], although it is conceivable that the quantum nanomechanics [46] of their sulfur-metal bond could be tuned by using chemical substitution strategies involving aromatic ring systems. Here, we close this gap by presenting a systematic study, based on plane wave pseudopotential density functional theory (DFT) calculations using the Perdew-Burke-Ernzerhof (PBE) exchange-correlation functional, of the thermal and mechanical desorption of two prototypical aliphatic thiolates, ethyl (Et-S) and butylthiolate (Bu-S), and a series of 3,5-disubstituted $p$-methyl thiophenolates (X-Ph-S in Table I) adsorbed on gold surfaces. In order to cope with different adsorption structures of thiolates on gold [29-31,47], we have considered two complementary models: a flat $\mathrm{Au}(111)$ surface and a defective surface with a vacancy at the adsorption site. See Supplemental Material (SM) [48] for computational details with references as well as validation of the PBE functional herein employed with respect to results obtained with the Heyd-ScuseriaErnzerhof (HSE) hybrid functional [48].

We shall first focus on the usual thermal desorption energies, i.e., $E_{\mathrm{des}}=E(\mathrm{RS})+E(\mathrm{Au})-E(\mathrm{RS}-\mathrm{Au})$ where $E(\mathrm{RS}-\mathrm{Au}), E(\mathrm{Au})$, and $E(\mathrm{RS})$ are the total electronic energy of optimized adsorbate system, clean surface, and

TABLE I. Thermal desorption energies $\left(E_{\text {des }}\right)$ and mechanical rupture force $\left(F_{\text {rup }}\right)$, see SM [48], for aliphatic and aromatic thiolates, see text, on flat and defective Au(111) surfaces. The font indicates if the mechanical desorption occurs through $\mathrm{Au}-\mathrm{Au}$ (bold) or $\mathrm{S}-\mathrm{Au}$ (italic) bond rupture. The values in parenthesis were obtained using the HSE hybrid density functional as detailed in the SM [48].

\begin{tabular}{lccccc}
\hline \hline \multirow{2}{*}{ Molecule } & \multicolumn{2}{c}{ Flat $\mathrm{Au}(111)$} & & \multicolumn{2}{c}{ Defective $\mathrm{Au}(111)$} \\
\cline { 1 - 2 } \cline { 5 - 6 } & $E_{\text {des }}(\mathrm{eV})$ & $F_{\text {rup }}(\mathrm{nN})$ & & $E_{\text {des }}(\mathrm{eV})$ & $F_{\text {rup }}(\mathrm{nN})$ \\
\hline $\mathrm{Bu}-\mathrm{S}$ & $\mathbf{2 . 1 5}$ & $\mathbf{1 . 6 8}$ & & $\mathbf{2 . 6 4}$ & $\mathbf{1 . 7 5}$ \\
$\mathrm{Et}-\mathrm{S}$ & $\mathbf{2 . 1 5}$ & $\mathbf{1 . 4 6}(\mathbf{1 . 4 1})$ & & $\mathbf{2 . 6 3}$ & $\mathbf{1 . 7 2}$ \\
$\mathrm{NH}_{2}-\mathrm{Ph}-\mathrm{S}$ & 1.74 & 2.25 & & $\mathbf{2 . 2 2}$ & $\mathbf{1 . 8 2}$ \\
$\mathrm{OCH}_{3}-\mathrm{Ph}-\mathrm{S}$ & 1.69 & 2.20 & & $\mathbf{2 . 1 5}$ & $\mathbf{1 . 8 2}$ \\
$\mathrm{H}-\mathrm{Ph}-\mathrm{S}$ & 1.69 & $2.21(2.34)$ & & $\mathbf{2 . 0 6}$ & $\mathbf{1 . 7 4}$ \\
$\mathrm{CN}-\mathrm{Ph}-\mathrm{S}$ & 1.69 & 2.11 & & $\mathbf{2 . 2 0}$ & $\mathbf{1 . 8 0}$ \\
$\mathrm{NO}_{2}-\mathrm{Ph}-\mathrm{S}$ & 1.68 & 2.32 & & $\mathbf{2 . 0 6}$ & $\mathbf{1 . 7 7}$ \\
\hline \hline
\end{tabular}


isolated thiolate, respectively. The $E_{\mathrm{des}}$ values of aliphatic vs aromatic thiols are vastly different on the flat gold surface (see Table I). Specifically, $E_{\mathrm{des}}$ of the aromatic thiols is on average about $20 \%$ lower than the aliphatic counterparts. Interestingly, this scenario still holds when the aromatic ring has different substituents (see SM for details [48]). Notwithstanding the higher thermal stability of aliphatic vs aromatic thiolates, mechanical desorption leads to easy detachment of aliphatic species whereas the rupture forces $\left(F_{\text {rup }}\right)$ required for aromatic thiolates are systematically higher; see Table I. This surprising finding can be explained by means of the energy profiles shown in Fig. 1 for the mechanically enforced detachment of aliphatic vs aromatic thiolates adsorbed on the flat $\mathrm{Au}(111)$ surface as a function of the detachment coordinate $D$; see SM for all profiles [48].

Two different classes of mechanical detachment scenarios are observed: For the aliphatic species, mechanical stretching of the thiolate-gold junction produces a gold chain consisting of two to three atoms which finally breaks at some $\mathrm{Au}-\mathrm{Au}$ bond [37,38], whereas for all aromatic thiolates, the force-induced rupture takes place at the $\mathrm{S}-\mathrm{Au}$ bond; see Table I. These different mechanical detachment mechanisms explain the dissimilar rupture forces, being $1.5 \mathrm{nN}$ for Au-Au cleavage in the aliphatic cases and $2.2 \mathrm{nN}$ for $\mathrm{S}-\mathrm{Au}$ cleavage in the aromatic cases. Therefore, aromatic thiolates are thermally less stable, but mechanically more stable toward desorption in comparison to the aliphatic analogues. This behavior is independent of the particular adsorption structure of the S-Au nanojunction itself, as demonstrated in the SM [48]. Moreover, we validated the performance of the semilocal PBE functional for computing the rupture forces of aliphatic vs aromatic thiolates by performing representative benchmark calculations using the hybrid HSE functional (see SM for background and computational details [48]). Most importantly,
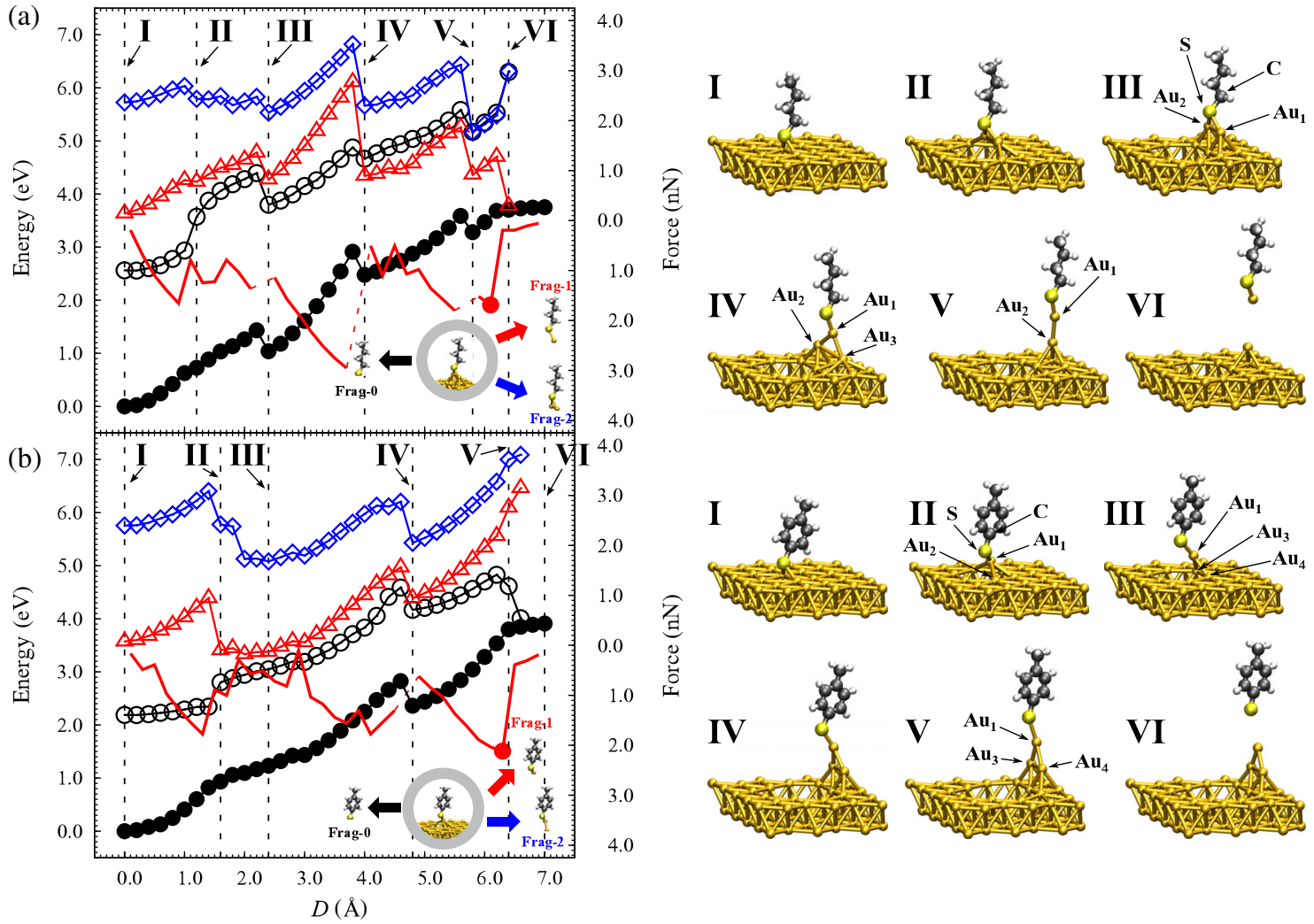

FIG. 1. Pathways and mechanisms of mechanical detachment of (a) aliphatic Bu-S and (b) aromatic $\mathrm{H}-\mathrm{Ph}-\mathrm{S}$ thiolates adsorbed on the flat $\mathrm{Au}(111)$ surface (see SM for all systems from Table I [48]). Left-hand panels: Total electronic energy along the preferred mechanical desorption pathway (filled black circles, left axis) and total electronic energies of the distinct vertical fragmentation products Frag-0 (empty black circles), Frag-1 (empty red triangles), and Frag-2 (empty blue diamonds), see text, as a function of the mechanical detachment coordinate $D$, see SM [48]. Force vs distance curves for regions of elastic deformation (solid red lines, right axis); the filled red circles indicate $F_{\text {rup }}$ as reported in Table I. Vertical dashed black lines indicate the position of the relevant structures I-VI, along detachment that are depicted in the right-hand panels. The inset depicts the fragmentation product (molecule only) for the different scenarios considered at $D=2.4 \AA$ for both systems. Right-hand panels: Configuration snapshots at important stages of mechanical desorption as defined in the left-hand panels at $D=0.0$ (I), 1.2 (II), 2.4 (III), 4.0 (IV), 5.8 (V), and 6.4 (VI) $\AA$ for Bu-S and at $D=0.0$ (I), 1.6 (II), 2.4 (III), 4.8 (IV), 6.4 (V), and 7.0 (VI) $\AA$ for H-Ph-S in the upper and lower part, respectively. Note that only the two uppermost layers of the surface slab are shown for clarity. 
the vastly different rupture forces of the aliphatic and aromatic reference systems agree with the HSE data each within $\pm 0.1 \mathrm{nN}$; see Table I.

In order to disclose the reasons behind these two different mechanical detachment mechanisms, "vertical fragmentation energies" $\left(E_{\text {frag }}\right)$ were calculated along the mechanical desorption pathway for Bu-S and $\mathrm{H}-\mathrm{Ph}-\mathrm{S}$ by considering different fragmentation scenarios in which either the $\mathrm{S}-\mathrm{Au}$ bonds or some selected $\mathrm{Au}-\mathrm{Au}$ bonds have been cleaved. These energies are defined via $E_{A-B}^{\mathrm{frag}}=E(A)+E(B)-E(A-B)$, where the fragments $A$ and $B$ are kept in the same configuration as in the nonfragmented reference structure $A-B$ (see SM for further details [48]). For H-Ph-S, see Fig. 1(b), the vertical fragmentation energy for the $\mathrm{S}-\mathrm{Au}$ bond $\left(E_{\text {frag }}^{0}\right)$ is always lower than for breaking a $\mathrm{Au}-\mathrm{Au}$ bond $\left(E_{\text {frag }}^{1}\right)$ and thus detaching a single sulfur-bound $\mathrm{Au}$ atom together with the thiolate. Notably, $E_{\text {frag }}^{0}$ decreases rapidly at the last elastic stage of the stretching pathway, i.e., between structures IV and $\mathrm{V}$, thus explaining the final $\mathrm{S}-\mathrm{Au}$ cleavage.

Interestingly, for Bu-S shown in Fig. 1(a), the fragmentation energy resulting from breaking the $\mathrm{S}-\mathrm{Au}$ bond, $E_{\text {frag }}^{0}$, lies initially below $E_{\text {frag }}^{1}$, until a significant plastic deformation at $D \approx 4.0 \AA$ suddenly rearranges the structure of the molecule or metal contact by means of a decrease of the coordination number of $\mathrm{S}$ with respect to the gold, caused by the breaking of the $\mathrm{Au}_{2}-\mathrm{S}$ bond (transition from structures III and IV). It is only after this plastic deformation that the cleavage of a $\mathrm{Au}-\mathrm{Au}$ bond becomes more favorable than the breakage of the S-Au contact, leading to a final aliphatic product in which an Au atom is extracted from the surface. Surprisingly, the arrangement of the $\mathrm{Bu}$ $\mathrm{S}$-Au junction at stage IV along the mechanical detachment pathway in Fig. 1(a) is very similar to the one found for the structure also labeled as IV in the aromatic pathway found for $\mathrm{H}-\mathrm{Ph}-\mathrm{S}$ [see Fig. 1(b)], where, however, the S-Au bond is broken instead. The fact that at this particular point $E_{\text {frag }}^{1}$ is smaller than $E_{\text {frag }}^{0}$ for the aliphatic ligand, whereas the reverse is true for the aromatic one, is an indication that it is the nature of the electronic structure of the carbon skeleton which has a fundamental influence on the S-Au interaction and, hence, on the mechanical vs thermal strength of the bond.

These astonishing findings can be rationalized in terms of the electronic structure of the two distinct adsorbate classes and its effect upon mechanically stretching these hybrid junctions. The evolution of the relative Löwdin charges (LC) for relevant atoms at the nanocontact and the Mulliken overlap population (MP) for relevant bonds along the mechanical desorption pathway of Bu-S and $\mathrm{H}-\mathrm{Ph}-\mathrm{S}$ initially adsorbed on a flat $\mathrm{Au}(111)$ surface are shown in
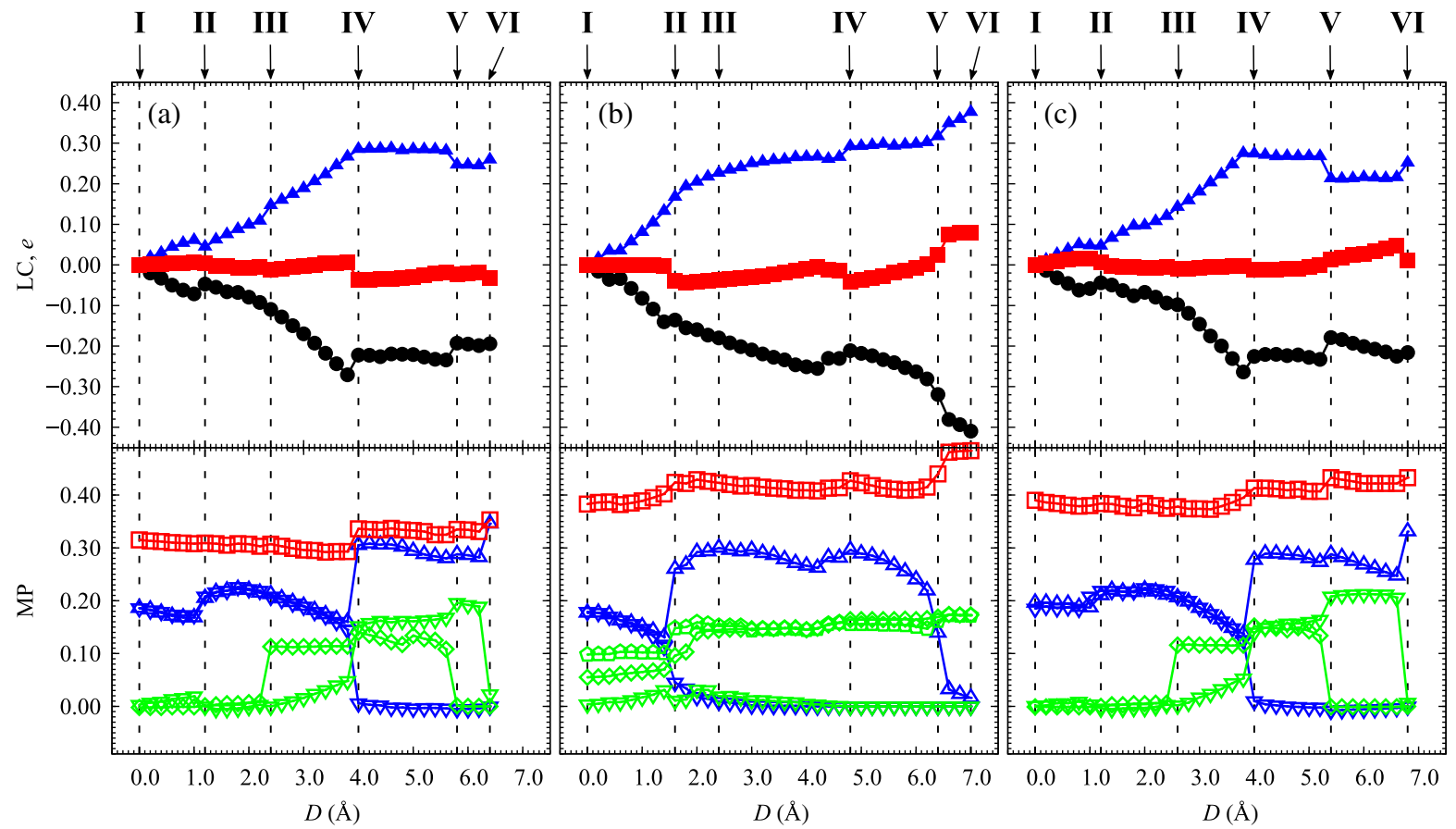

FIG. 2. Evolution of relative Löwdin charges (LC) and Mulliken overlap population (MP) along the mechanical detachment pathways for (a) $\mathrm{Bu}-\mathrm{S}$ and (b) $\mathrm{H}-\mathrm{Ph}-\mathrm{S}$ adsorbed on the flat $\mathrm{Au}(111)$ surface as well as for (c) $\mathrm{H}-\mathrm{Ph}-\mathrm{S}$ adsorbed on a defective surface. Upper panels: LC with respect to the initial structure at $D=0.0 \AA$ for the anchoring $S$ atom (filled black circles), the Au slab (filled blue up triangles), and the alkyl-aryl moiety $R$ (filled red squares). Lower panels: MP for relevant bonds along the different pathways, namely, $\mathrm{S}-\mathrm{C}$ (empty red squares), $\mathrm{S}-\mathrm{Au}_{1}$ (empty blue up triangles), $\mathrm{S}-\mathrm{Au}_{2}$ (empty blue down triangles), $\mathrm{Au}_{1}-\mathrm{Au}_{2}$ (empty green down triangles), $\mathrm{Au}_{1}-\mathrm{Au}_{3}$ (empty green diamonds), and $\mathrm{Au}_{1}-\mathrm{Au}_{4}$ (empty green pentagons); see right-hand panels in Fig. 1 for $\mathrm{Au} i$ atom labeling. 
Fig. 2. The major differences in the mechanical behavior of aliphatic vs aromatic thiolates occur in the transition from structures I $\rightarrow$ III and again from IV $\rightarrow$ V. For both species, charge is being reshuffled from the gold surface to the molecular moiety from I to III, which is mostly accumulated on the anchoring $\mathrm{S}$ atom as a result of stretching. For $\mathrm{Bu}-\mathrm{S}$, the $\mathrm{Au}-\mathrm{S}$ bonds are slightly weakened from I $\rightarrow$ II, where two Au atoms are lifted from the surface to finally produce structure III, in which there are two adatoms on the surface [see Fig. 1(a)]. For H-Ph-S, in turn, the mechanical force induces much larger charge flow from the Au surface to the anchoring $\mathrm{S}$ atom, resulting in a weakening of the covalent character of the $\mathrm{Au}-\mathrm{S}$ bonds, as measured by the MP, thus leading to the breakage of one of the $\mathrm{S}-\mathrm{Au}$ bonds, as illustrated by structure II in Fig. 1(b). At this stage, a concomitant strengthening of the $\mathrm{S}-\mathrm{C}$ bond is also observed.

An even more contrasting behavior is observed in the stretching of structures IV $\rightarrow \mathrm{V}$. At this later stage there is an increasing charge reshuffling from the $\mathrm{R}$ group to the anchoring $\mathrm{S}$ atom for the aromatic thiolate. This causes a strengthening of the $\mathrm{S}-\mathrm{C}$ bond, as evidenced by the MP analysis, and a concomitant weakening of the $\mathrm{Au}-\mathrm{S}$ bond, even lower than the $\mathrm{Au}-\mathrm{Au}$ bonds, thus causing the final $\mathrm{S}-\mathrm{Au}$ bond rupture. In contrast, for the aliphatic molecule this charge flow is not found and, hence, the $\mathrm{Au}-\mathrm{Au}$ bonds are weaker than the $\mathrm{S}-\mathrm{Au}$ bond in the entire range of stretching. For this reason, the later stages of its mechanical detachment involve only $\mathrm{Au}-\mathrm{Au}$ bond rupture events.

In short, the different mechanochemical response of aliphatic vs aromatic thiolates seems to be related to the enhanced capacity of the "aromatic S atom" to bear a negative charge. This, in turn, is associated with a good overlap of the $p$ orbitals of this atom with the $\mathrm{C}(p)$ orbitals in the aromatic ring, as evidenced by the projected density of states analysis of Fig. 3, which shows that there is a good overlap between $\mathrm{S}(p)$ states with $\mathrm{C}(p)$ states about $1 \mathrm{eV}$ below the Fermi level for the thiophenolate H-Ph-S. This is not the case for aliphatic analogues, such as $\mathrm{Bu}-\mathrm{S}$, due to the lack of an aromatic ring system. In fact, for $\mathrm{H}-\mathrm{Ph}-\mathrm{S}$ adsorbed on the flat Au surface, these $\mathrm{S}(p)-\mathrm{C}(p)$ states are perfectly aligned with the $\mathrm{Au}(d)$ orbitals of the gold atoms to which the $\mathrm{S}$ atom is anchored (indicated with an arrow in Fig. 3). This is supported by electron density difference analysis (see insets in Fig. 3), which demonstrates that most of the charge is accumulated between the $\mathrm{S}$ and $\mathrm{Au}$ atoms in the case of aliphatic Bu-S, while some charge is also accumulated on a $p$ orbital of the $\mathrm{C}$ atom directly attached to the anchoring $\mathrm{S}$ atom for the aromatic $\mathrm{H}-\mathrm{Ph}-\mathrm{S}$ species. Moreover, Fig. 2 in the SM not only provides further evidence for this very bonding scenario and thus the qualitative difference of aromatic vs aliphatic thiolates attached to gold surfaces, but also reveals in addition that the $\mathrm{S}(s)$ orbitals have a negligible participation in the bonding states close to the Fermi level [48].

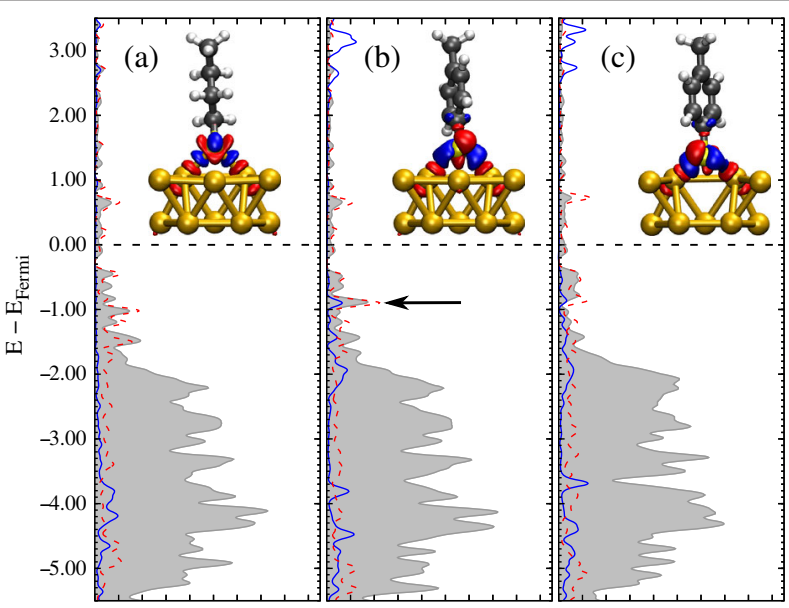

FIG. 3. Projected density of states for the $\mathrm{S}(p)$ orbital (broken red line) and $\mathrm{C}(p)$ orbital (continuous blue line) at the anchoring $\mathrm{S}$ atom and the $\mathrm{C}$ atom attached to it, respectively, and for the $\mathrm{Au}(d)$ orbitals (shaded gray area) at those Au atoms to which the molecule is bonded to corresponding to (a) Bu-S and (b) H-Ph-S adsorbed on the flat $\mathrm{Au}(111)$ surface as well as for (c) H-Ph-S adsorbed on a defective surface, all shown at $D=0.0 \AA$. For the case of $\mathrm{H}-\mathrm{Ph}-\mathrm{S}$ on the flat surface, the arrow indicates an important electronic state in which the orbitals of S, C, and $\mathrm{Au}$ atoms overlap. The insets in the upper part illustrate the corresponding electron density difference isosurfaces at an isovalue of $0.005 e / \AA^{3}$ for the fragmentation of the S-Au bond; blue (red) isosurfaces indicate charge accumulation (depletion).

Let us finally address the impact of a vacancy defect in the vicinity of the adsorption site on the gold surface. The mechanical detachment mechanism remains unaltered for the aliphatic thiolates, $F_{\text {rup }}$ being only slightly larger. In stark contrast, adsorption at a defect has two contraposing effects for aromatic junctions; while the junction is thermally stronger (i.e., higher $E_{\text {des }}$ ), it becomes mechanically weaker (i.e., lower $F_{\text {rup }}$ ) according to Table I. Remarkably, the mechanical desorption pathway (see SM [48]) for the aromatic thiolates on the defective surface is identical to that displayed by the aliphatic analogues; i.e., the final rupture of the hybrid nanojunction takes place at a $\mathrm{Au}-\mathrm{Au}$ bond. This surprising behavior can be rationalized on the basis of the resemblance between the stress-induced evolution of electronic structures of the junction in both cases [compare Figs. 2(a) and 2(c)]. This resemblance, in turn, originates from a shift upwards in energy of the $\mathrm{Au}(d)$ states due to the vacancy, as a result of which the overlap of the $\mathrm{S}(p)-\mathrm{C}(p)$ state is disrupted, thereby rendering the bonding pattern of the aromatic thiolates on the defective surface similar to that of the aliphatic analogues on the flat $\mathrm{Au}(111)$ surface.

In conclusion, we disclosed the counterintuitive phenomenon that, although aliphatic thiolates are more strongly bound to flat $\mathrm{Au}(111)$ gold surfaces than aromatic species in terms of the thermal desorption energy, they are more easily mechanically desorbed upon applying 
mechanical forces. This is because the two classes of thiolates give rise to different intermediate nanostructures in response to mechanical stress and also to different bond breaking scenarios just before mechanical detachment. These observations can be directly related to qualitative differences in chemical bonding right at the molecule-metal nanojunction. The presence of defects in the surface significantly modifies the thermal and mechanical stability of the aromatic thiolate-gold nanojunctions. Specifically, a vacancy defect results in enhanced binding energies while the mechanical stabilities are reduced.

Beyond the specific case, our work demonstrates that knowing the thermal desorption energy of molecule or metal contacts does not help to predict their mechanical strength, which has important consequences on assessing the stability of molecular coatings toward friction and wear. Furthermore, their unveiled different mechanical response depending on the aliphatic or aromatic nature of the thiolate and on the type of gold surface demonstrates that it is possible to tailor the mechanical strength of $\mathrm{Au}-\mathrm{S}$ contacts and to exert control on which nanostructures are formed when these contacts are subjected to mechanical stress. These are certainly appealing features for the rational design of $\mathrm{Au}-\mathrm{S}$ contacts with practical applications in many fields.

M.E.Z. M. and D. M. gratefully acknowledge financial support by DAAD (Fellowship) and DFG (Reinhart Koselleck Grant No. MA 1547/9), respectively. The simulations were carried out at HLRS (Stuttgart), BOVILAB@RUB (Bochum), and RV-NRW.

*Present address: INFIQC-CONICET, Departamento de Química Teórica y Computacional, Facultad de Ciencias Químicas, Univesidad Nacional de Córdoba, Córdoba, Argentina.

martin.zoloff@theochem.ruhr-uni-bochum.de

†Present address: Departament de Ciència de Materials i Química Física and IQTCUB, Universitat de Barcelona, Av. Diagonal 645, 08028 Barcelona, Spain.

[1] A. Dhakshinamoorthy, A. M. Asiri, and H. Garcia, ACS Catal. 7, 2896 (2017).

[2] M. L. Personick, R. J. Madix, and C. M. Friend, ACS Catal. 7, 965 (2017).

[3] E. Gross, J. H. -C. Liu, F. D. Toste, and G. A. Somorjai, Nat. Chem. 4, 947 (2012).

[4] W.-Y. Lo, N. Zhang, Z. Cai, L. Li, and L. Yu, Acc. Chem. Res. 49, 1852 (2016).

[5] C. Huang, S. Chen, K. Baruël Ørnso, D. Reber, M. Baghernejad, Y. Fu, T. Wandlowski, S. Decurtins, W. Hong, K. S. Thygesen, and S.-X. Liu, Angew. Chem. Int. Ed. 54, 14304 (2015).

[6] T. A. Su, H. Li, M. L. Steigerwald, L. Venkataraman, and C. Nuckolls, Nat. Chem. 7, 215 (2015).

[7] A. Saffarzadeh, F. Demir, and G. Kirczenow, Phys. Rev. B 89, 045431 (2014).
[8] M.-J. Huang, L.-Y. Hsu, M.-D. Fu, S.-T. Chuang, F.-W. Tien, and C.-h. Chen, J. Am. Chem. Soc. 136, 1832 (2014).

[9] F. Benz, M. K. Schmidt, A. Dreismann, R. Chikkaraddy, Y. Zhang, A. Demetriadou, C. Carnegie, H. Ohadi, B. de Nijs, R. Esteban, J. Aizpurua, and J. J. Baumberg, Science 354, 726 (2016).

[10] J. Li, J. Liu, and C. Chen, ACS Nano 11, 2403 (2017).

[11] H. Qiu, Y. Min, Z. Rodgers, L. Zhang, and A. Z. Wang, WIREs Nanomed. Nanobiotechnol. 9, e1456 (2017).

[12] X. Chen, M. Strange, and H. Häkkinen, Phys. Rev. B 85, 085422 (2012).

[13] L. Dykman and N. Khlebtsov, Chem. Soc. Rev. 41, 2256 (2012).

[14] E. C. Dreaden, A. M. Alkilany, X. Huang, C. J. Murphy, and M. A. El-Sayed, Chem. Soc. Rev. 41, 2740 (2012).

[15] D. Giljohann, D. Seferos, W. Daniel, M. Massich, P. Patel, and C. Mirkin, Angew. Chem., Int. Ed. Engl. 49, 3280 (2010).

[16] V. Y. Kutsenko, Y. Y. Lopatina, L. Bossard-Giannesini, O. A. Marchenko, O. Pluchery, and S. V. Snegir, Nanotechnology 28, 235603 (2017).

[17] C. Vericat, M. E. Vela, G. Benitez, P. Carro, and R. C. Salvarezza, Chem. Soc. Rev. 39, 1805 (2010).

[18] P. Zhao, N. Li, and D. Astruc, Coord. Chem. Rev. 257, 638 (2013).

[19] H. B. Akkerman and B. de Boer, J. Phys. Condens. Matter 20, 013001 (2008).

[20] N. J. Tao, Nat. Nanotechnol. 1, 173 (2006).

[21] C. Zeng, Y. Chen, A. Das, and R. Jin, J. Phys. Chem. Lett. 6, 2976 (2015).

[22] Y. Chen, C. Liu, Q. Tang, C. Zeng, T. Higaki, A. Das, D.-e. Jiang, N. L. Rosi, and R. Jin, J. Am. Chem. Soc. 138, 1482 (2016).

[23] T. Higaki, C. Zeng, Y. Chen, E. Hussain, and R. Jin, CrystEngComm 18, 6979 (2016).

[24] W. Kurashige, Y. Niihori, S. Sharma, and Y. Negishi, Coord. Chem. Rev. 320-321, 238 (2016).

[25] D. M. Chevrier, R. Yang, A. Chatt, and P. Zhang, Nanotechnol. Rev. 4, 193 (2015).

[26] W. Kurashige, Y. Niihori, S. Sharma, and Y. Negishi, J. Phys. Chem. Lett. 5, 4134 (2014).

[27] C. Zeng, H. Qian, T. Li, G. Li, N. L. Rosi, B. Yoon, R. N. Barnett, R. L. Whetten, U. Landman, and R. Jin, Angew. Chem. Int. Ed. 51, 13114 (2012).

[28] M. Konôpka, R. Rousseau, I. Štich, and D. Marx, J. Am. Chem. Soc. 126, 12103 (2004).

[29] E. Pensa, E. Cortés, G. Corthey, P. Carro, C. Vericat, M. H. Fonticelli, G. Benítez, A. A. Rubert, and R. C. Salvarezza, Acc. Chem. Res. 45, 1183 (2012).

[30] H. Häkkinen, Nat. Chem. 4, 443 (2012).

[31] T. Bürgi, Nanoscale 7, 15553 (2015).

[32] Y. Xue, X. Li, H. Li, and W. Zhang, Nat. Commun. 5, 4348 (2014).

[33] J. Ribas-Arino and D. Marx, Chem. Rev. 112, 5412 (2012).

[34] M. E. Zoloff Michoff, J. Ribas-Arino, and D. Marx, Phys. Rev. Lett. 114, 075501 (2015).

[35] P. Seema, J. Behler, and D. Marx, Phys. Rev. Lett. 115, 036102 (2015).

[36] M. Konôpka, R. Turanský, J. Reichert, H. Fuchs, D. Marx, and I. Štich, Phys. Rev. Lett. 100, 115503 (2008). 
[37] D. Krüger, R. Rousseau, H. Fuchs, and D. Marx, Angew. Chem. Int. Ed. 42, 2251 (2003).

[38] D. Krüger, H. Fuchs, R. Rousseau, D. Marx, and M. Parrinello, Phys. Rev. Lett. 89, 186402 (2002).

[39] E. Sabatini, J. Cohen-Boulakia, M. Bruening, and I. Rubinstein, Langmuir 9, 2974 (1993).

[40] S.-C. Chang, I. Chao, and Y.-T. Tao, J. Am. Chem. Soc. 116, 6792 (1994).

[41] L. Romaner, G. Heimel, M. Gruber, J.-L. Brédas, and E. Zojer, Small 2, 1468 (2006).

[42] R. Turanský, M. Konôpka, N. L. Doltsinis, I. Štich, and D. Marx, Phys. Chem. Chem. Phys. 12, 13922 (2010).

[43] W. Hong, D. Z. Manrique, P. Moreno-Garcia, M. Gulcur, A. Mishchenko, C. J. Lambert, M. R. Bryce, and T. Wandlowski, J. Am. Chem. Soc. 134, 2292 (2012).

[44] W. R. French, C. R. Iacovella, I. Rungger, A. M. Souza, S. Sanvito, and P. T. Cummings, J. Phys. Chem. Lett. 4, 887 (2013).

[45] M. E. Zoloff Michoff, M. Ezequiel Castillo, and E. P. M. Leiva, J. Phys. Chem. C 119, 5090 (2015).

[46] T. Stauch and A. Dreuw, Chem. Rev. 116, 14137 (2016).

[47] Y. Pei and X. C. Zeng, Nanoscale 4, 4054 (2012).

[48] See Supplemental Material at http://link.aps.org/ supplemental/10.1103/PhysRevLett.122.086801, which includes Refs. [49-62], for a detailed account of the computational methods employed in this work, the validation of the GGA functional employed, and additional results for the systems not shown in the main text.
[49] J. P. Perdew, K. Burke, and M. Ernzerhof, Phys. Rev. Lett. 77, 3865 (1996).

[50] P. Giannozzi et al., J. Phys. Condens. Matter 21, 395502 (2009).

[51] D. Vanderbilt, Phys. Rev. B 41, 7892 (1990).

[52] N. Troullier and J. L. Martins, Phys. Rev. B 43, 1993 (1991).

[53] N. Troullier and J. L. Martins, Phys. Rev. B 43, 8861 (1991).

[54] E. Artacho, E. Anglada, O. Diéguez, J. D. Gale, A. García, J. Junquera, R. M. Martin, P. Ordejón, J. M. Pruneda, D. Sánchez-Portal, and J. M. Soler, J. Phys. Condens. Matter 20, 064208 (2008).

[55] P. Maksymovych, D. C. Sorescu, and J. T. Yates, J. Phys. Chem. B 110, 21161 (2006).

[56] J. Nara, S. Higai, Y. Morikawa, and T. Ohno, J. Chem. Phys. 120, 6705 (2004).

[57] J. Heyd, G. E. Scuseria, and M. Ernzerhof, J. Chem. Phys. 118, 8207 (2003).

[58] J. Heyd, G. E. Scuseria, and M. Ernzerhof, J. Chem. Phys. 124, 219906 (2006).

[59] C. A. Szafranski, W. Tanner, P. E. Laibinis, and R. L. Garrell, Langmuir 14, 3570 (1998).

[60] L.-J. Wan, M. Terashima, H. Noda, and M. Osawa, J. Phys. Chem. B 104, 3563 (2000).

[61] D. Käfer, A. Bashir, and G. Witte, J. Phys. Chem. C 111, 10546 (2007).

[62] S. Miranda-Rojas, A. Muñoz Castro, R. Arratia-Pérez, and F. Mendizábal, Phys. Chem. Chem. Phys. 15, 20363 (2013). 